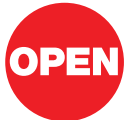

SUBJECT AREAS:

CHEMICAL SYNTHESIS

METHODS

NANOPARTICLES

NANOTECHNOLOGY

Received

3 May 2011

Accepted

30 June 2011

Published

14 July 2011

Correspondence and requests for materials should be addressed to Y.L. (ydli@mail. tsinghua.edu.cn)

\section{General preparation for Pt-based alloy nanoporous nanoparticles as potential nanocatalysts}

\author{
Dingsheng Wang, Peng Zhao \& Yadong Li
}

Department of Chemistry, Tsinghua University, Beijing 100084, P. R. China.

Although Raney nickel made by dealloying has been used as a heterogeneous catalyst in a variety of organic syntheses for more than $\mathbf{8 0}$ years, only recently scientists have begun to realize that dealloying can generate nanoporous alloys with extraordinary structural characteristics. Herein, we achieved successful synthesis of a variety of monodisperse alloy nanoporous nanoparticles via a facile chemical dealloying process using nanocrystalline alloys as precursors. The as-prepared alloy nanoporous nanoparticles with large surface area and small pores show superior catalytic properties compared with alloyed nanoparticles. It is believed that these novel alloy nanoporous nanoparticles would open up new opportunities for catalytic applications.

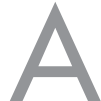

s early as 1926, American engineer Murray Raney developed an alternative catalyst for the hydrogenation of vegetable oils in industrial processes: Raney nickel..$^{1}$ It was produced via selective leaching of a block of nickel-aluminium alloy $\left(\mathrm{NiAl}_{3}\right.$ and $\mathrm{Ni}_{2} \mathrm{Al}_{3}$ ) with concentrated sodium hydroxide. This treatment dissolved most of the aluminium out of the alloy, leading to the formation of porous nickel-aluminium alloy (NiAl) with large surface area. These porous alloys made by dealloying were found to show high catalytic activity and have been used as a heterogeneous catalyst in a variety of organic syntheses for more than 80 years. This typical example confirms that it is very significant to endow alloys with large surface area for their catalytic applications. However, only recently scientists have begun to realize that dealloying can generate nanoporous alloys with large surface area.

Considering the limited resource of noble metals, alloys are emerging as ideal candidate as low-cost catalysts in the field of industrial catalysis that can replace noble metal catalysts. ${ }^{2-5}$ But in the early years, alloys obtained by traditional metallurgical techniques possessed very low surface area, leading to the limitation of their catalytic applications. ${ }^{6}$ Nowadays, the development of nanotechnology makes it possible to synthesize nanocrystalline alloys with small particle size and large surface area. ${ }^{7-10}$ However, it is still not an easy task to prepare monodisperse alloy nanoporous nanoparticles with pore size distributions centered below $5 \mathrm{~nm} .{ }^{11}$ Such alloy nanoporous nanoparticles possess not only large surface area but also unique structural properties. ${ }^{12-14}$ Therefore, they are more promising in catalytic applications than alloyed nanoparticles.

Only a few strategies have been developed to prepare nanoporous alloys. ${ }^{15-22}$ The most comment method is templated growth, that is, synthesis of alloys in sacrificial porous molds. ${ }^{23-26}$ For example, mesoporous $\mathrm{SiO}_{2}{ }^{24}$ and carbon $^{25}$ were used as molds for preparation of $\mathrm{Au}-\mathrm{Cu}$ alloy nanoparticles and PdCo@Pd core-shell nanoparticles respectively. Xu and co-workers ${ }^{26}$ successfully fabricated $\mathrm{Au} @ \mathrm{Ag}$ core-shell nanoparticles immobilized on a metal-organic framework (MOF). A dealloying method is another way in generating porous alloys. ${ }^{27}$ Dealloying is the selective dissolution of the less-noble component from alloy in form of bulk or thin film, leaving a nanoporous residue. ${ }^{28-30}$ It contains electrochemical dealloying and chemical dealloying. In comparison with the former, the chemical dealloying is a free corrosion way, which is much easier to implement. Raney nickel is just a successful example of porous alloy obtained by chemical dealloying. ${ }^{1}$ However, chemical dealloying has thus far not been recognized as an effective method to prepare useful heterogeneous catalysts like Raney nickel.

Very recently, we developed an effective noble-metal-induced-reduction (NMIR) method to synthesize bimetallic nanocrystals. ${ }^{31-33}$ Via this strategy, synthesis of nanocrystalline Au-, Pd-, Pt-, Ir-, Ru-, Rh-based intermetallics and alloys with small particle size and large surface area can be easily achieved. Herein, we demonstrate that monodisperse alloy nanoporous nanoparticles can be prepared by selective dissolution of the less-noble component from as-synthesized nanocrystalline intermetallics and alloys. Because the precursor itself has small particle size and large surface area, the as-obtained dealloyed product has not only small pore 
size below $5 \mathrm{~nm}$, but also much larger surface area. Thus, the alloy nanoporous nanoparticles are considered to be potential candidate as nanocatalysts.

\section{Results}

We employ preparation of Pt-Ni nanoporous nanoparticles as an example to introduce this chemical dealloying method using nanocrystalline alloys as precursors. In a typical procedure, $\mathrm{Pt}-\mathrm{Ni}$ alloyed nanoparticles were firstly synthesized in hot octadecylamine (ODA) solvent using $\mathrm{H}_{2} \mathrm{PtCl}_{6} \cdot 6 \mathrm{H}_{2} \mathrm{O}$ and $\mathrm{Ni}\left(\mathrm{NO}_{3}\right)_{2} \cdot 6 \mathrm{H}_{2} \mathrm{O}$ as the starting materials. Then, an excess amount of concentrated nitric acid was introduced as an oxidant to etch away Ni from Pt-Ni nanoparticles (see details in Experimental Section). Figure 1 shows transmission electron microscope (TEM) and high-resolution TEM (HRTEM) images of as-obtained Pt-Ni nanoparticles and corresponding dealloyed products. It can be seen that $\mathrm{Pt}-\mathrm{Ni}$ nanoparticles have an average size of $20 \mathrm{~nm}$ and polygonal morphology with clear edges and corners (Fig. 1a). After corrosion, the edges and corners of particles disappear and the particle size decreases to about $15 \mathrm{~nm}$. It is also obvious that the dealloyed nanoparticles are porous (Fig. 1c). Powder X-ray diffraction (XRD) measurements were used to identify the internal crystalline structures of as-prepared products. We compare the XRD results of Pt-Ni nanoparticles and dealloyed Pt-Ni and find that, with the dealloying of $\mathrm{Pt}-\mathrm{Ni}$, all the peaks are shifted slightly to smaller $2 \theta$ values, indicative of increased d-spacing and expansion of the lattice constant, due to the deviation of the smaller $\mathrm{Ni}$ atoms from the Pt fcc lattice (Supporting Information, Fig. S1). Chemical composition of the samples was further checked by energy dispersive spectroscopy (EDS). From the spectra shown in Figure S2 (Supporting Information), we can see that the compositions of $\mathrm{Pt}-\mathrm{Ni}$ nanoparticles and nanoporous $\mathrm{Pt}-\mathrm{Ni}$ are nearly $\mathrm{Pt}_{0.36} \mathrm{Ni}_{0.64}$ and $\mathrm{Pt}_{0.79} \mathrm{Ni}_{0.21}$ respectively. About $85 \%$ of $\mathrm{Ni}$ has been etched away. Two contrast experiments were carried out to confirm that concentrated nitric acid couldn't completely remove Ni atoms from $\mathrm{Pt}-\mathrm{Ni}$ alloys. The reaction between nitric acid and $\mathrm{Ni}$ is very fast and can finish within $1 \mathrm{~min}$. When the reaction time was prolonged to $10 \mathrm{~min}$, no changes could be observed. Additionally, when the asprepared nanoporous $\mathrm{Pt}-\mathrm{Ni}$ alloys were added to fresh concentrated nitric acid, no more $\mathrm{Ni}$ could be removed. The incompleteness is because the surfaces are fully covered by the inert Pt which leads to surface passivation. This result is also in accordance with Pickering's viewpoint that it is common to observe a significant residue of the less-noble metal in the dealloyed material. ${ }^{34}$

\section{Discussion}

The chemical dealloying treatment endows Pt-Ni alloys with some advantageous material properties: large surface area and small pores, which are desired in their catalytic applications. Brunauer-EmmettTeller (BET) measurements were used to compare the surface areas of Pt-Ni nanoparticles and dealloyed Pt-Ni. The data of $\mathrm{N}_{2}$ adsorption/desorption analysis are shown in Figure S3 (Supporting Information). The results confirm that the surface areas of Pt-Ni nanoparticles and dealloyed $\mathrm{Pt}-\mathrm{Ni}$ are 35.3 and $60.1 \mathrm{~m}^{2} \mathrm{~g}^{-1}$ respectively. It is obvious that the surface area of $\mathrm{Pt}-\mathrm{Ni}$ alloys increases greatly after corrosion. More importantly, the Pt-Ni alloys after dealloying treatment exhibit porous characteristic. The Barrett-JoynerHalenda (BJH) pore-size distribution curve (Fig. 2) indicates that the as-obtained dealloyed $\mathrm{Pt}-\mathrm{Ni}$ shows a narrow pore-size distribution, and the average pore diameter is $2 \mathrm{~nm}$. Compared with particulate systems, nanoporous $\mathrm{Pt}-\mathrm{Ni}$ alloys with pore size distribution centered $2 \mathrm{~nm}$ hold a great advantage that all the surface areas of nanoporous Pt-Ni are electrically accessible. So, this new class of
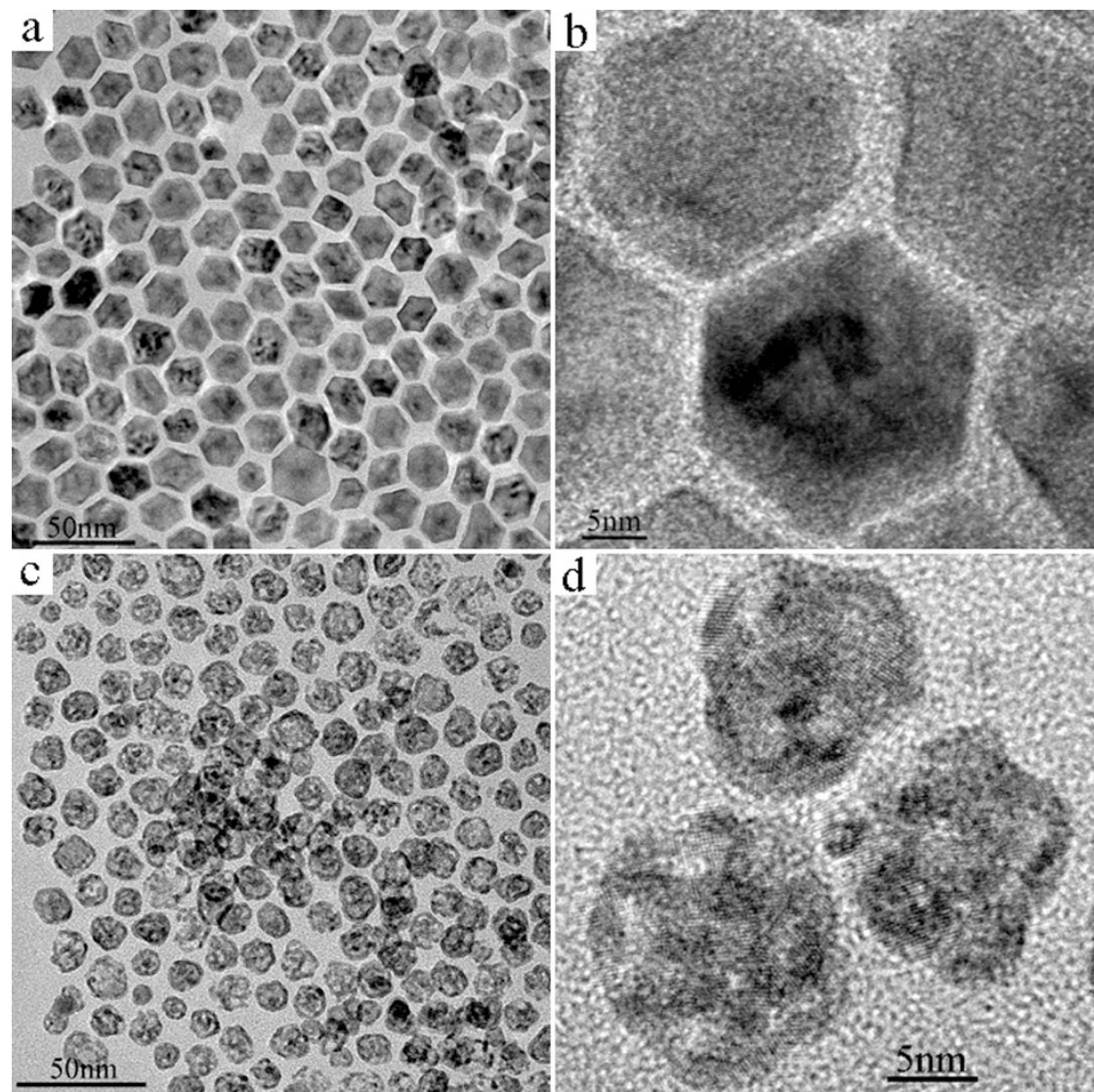

Figure 1 Nanoalloys observed by TEM and HRTEM. (a) TEM image of Pt-Ni nanoparticles. (b) HRTEM image of Pt-Ni nanoparticles. (c) TEM image of nanoporous Pt-Ni alloys. (d) HRTEM image of nanoporous Pt-Ni alloys. 


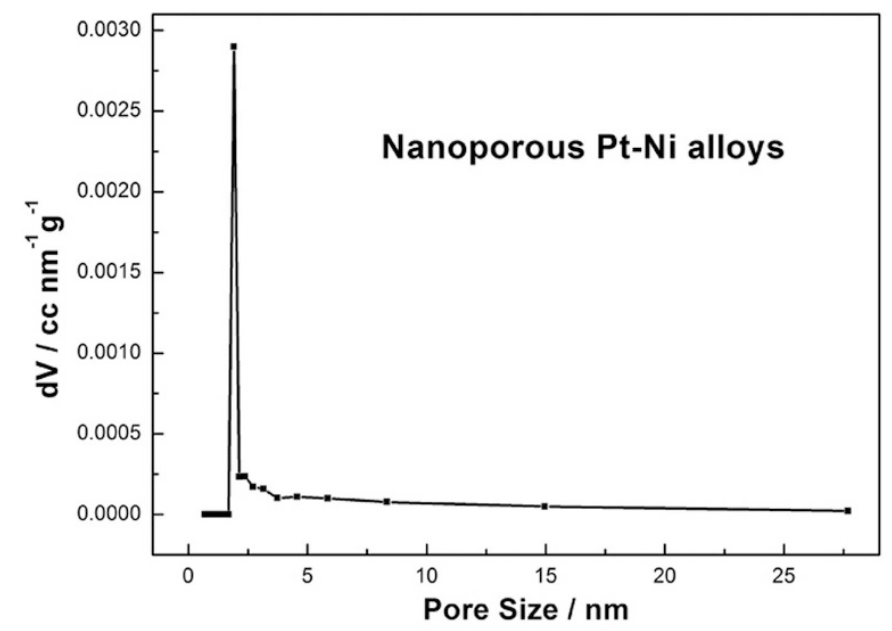

Figure $2 \mid$ Porous characteristic of alloys. BJH pore-size distribution curve of nanoporous Pt-Ni alloys.

nanomaterials with extraordinary structural characteristics represents nearly ideal electrodes for electrocatalysis.

In order to illuminate superior catalytic properties and potential applications of as-synthesized Pt-Ni nanoporous nanoparticles, the comparison of electrochemical properties of Pt-Ni nanoparticles and nanoporous $\mathrm{Pt}-\mathrm{Ni}$ was performed. The measurements were conducted by using a three-compartment electrochemical cell (see details in Experimental Section) and the results were summarized in Figure 3. Introducing Pt-Ni nanoparticles and nanoporous Pt-Ni as the active materials respectively, we recorded their cyclic voltammetries (CVs) at room temperature in $0.1 \mathrm{M} \mathrm{HClO}_{4}$ solutions. As shown in Figure $3 \mathrm{a}$, the CVs exhibit typical Pt-like $\mathrm{H}_{\text {upd }}$ features which contain two distinctive potential regions associated with $\mathrm{H}_{\text {upd }}$ adsorption/desorption process and the formation of an $\mathrm{OH}_{\mathrm{ad}}$ layer. Based on the CV curves, we calculated the electrochemically active surface area (ECSA) of catalysts by measuring the charge collected in the $\mathrm{H}_{\text {upd }}$ adsorption/desorption region. The calculation results show that the specific ECSAs (the ECSA per unit weight of $\mathrm{Pt}$ ) of $\mathrm{Pt}-\mathrm{Ni}$ nanoparticles and nanoporous $\mathrm{Pt}-\mathrm{Ni}$ are 33.7 and $85.6 \mathrm{~m}^{2} / \mathrm{g}_{\mathrm{Pt}}$ respectively (Fig. $3 \mathrm{~b}$ ). It is clear that $\mathrm{Pt}-\mathrm{Ni}$ nanoporous nanoparticles display about 2.5-times larger specific ECSA than Pt-Ni nanoparticles. We also note that the specific ECSA of nanoporous $\mathrm{Pt}-\mathrm{Ni}$ is larger than that of commercial $\mathrm{Pt} / \mathrm{C}$ catalyst reported in the literature ${ }^{5}$. It must be stated that, here we just provide a primary example to confirm the superiority of nanoporous alloys and their potential in catalytic applications, there is still plenty of room to further explore their rich functionalities in various fields including electrochemical catalysis, heterogeneous catalysis, and semi-homogeneous catalysis. The large surface area of alloy nanoporous nanoparticles can facilitate access of reactant molecules to catalysts, which ensures high activity of catalysts; while their small pores can differentiate reactant molecules with different size and structure, which ensures high selectivity of catalysts. Therefore, this new type of alloy nanoporous nanoparticles provides great opportunities for discovery of novel and effective nanocatalysts. Although the nanoporous alloys fabricated by the dealloying method have been widely reported recently, for example, Shao and co-workers ${ }^{35}$ demonstrated the fabrication of porous/hollow $\mathrm{Pd}-\mathrm{Cu}$ nanoparticles by selectively dissolving a less noble metal $(\mathrm{Cu})$ using an electrochemical dealloying process; Dutta and co-workers ${ }^{36}$ successfully prepared a highly active $\mathrm{PtCu}$ dealloyed catalyst via partial removal of the copper by acid leaching, our methodology has its own characteristics: highly monodispersed alloy nanocrystals are used as precursors and monodisperse alloy nanoporous nanoparticles can be obtained. Considering that our prepared monodisperse nanoporous nanoparticles can be
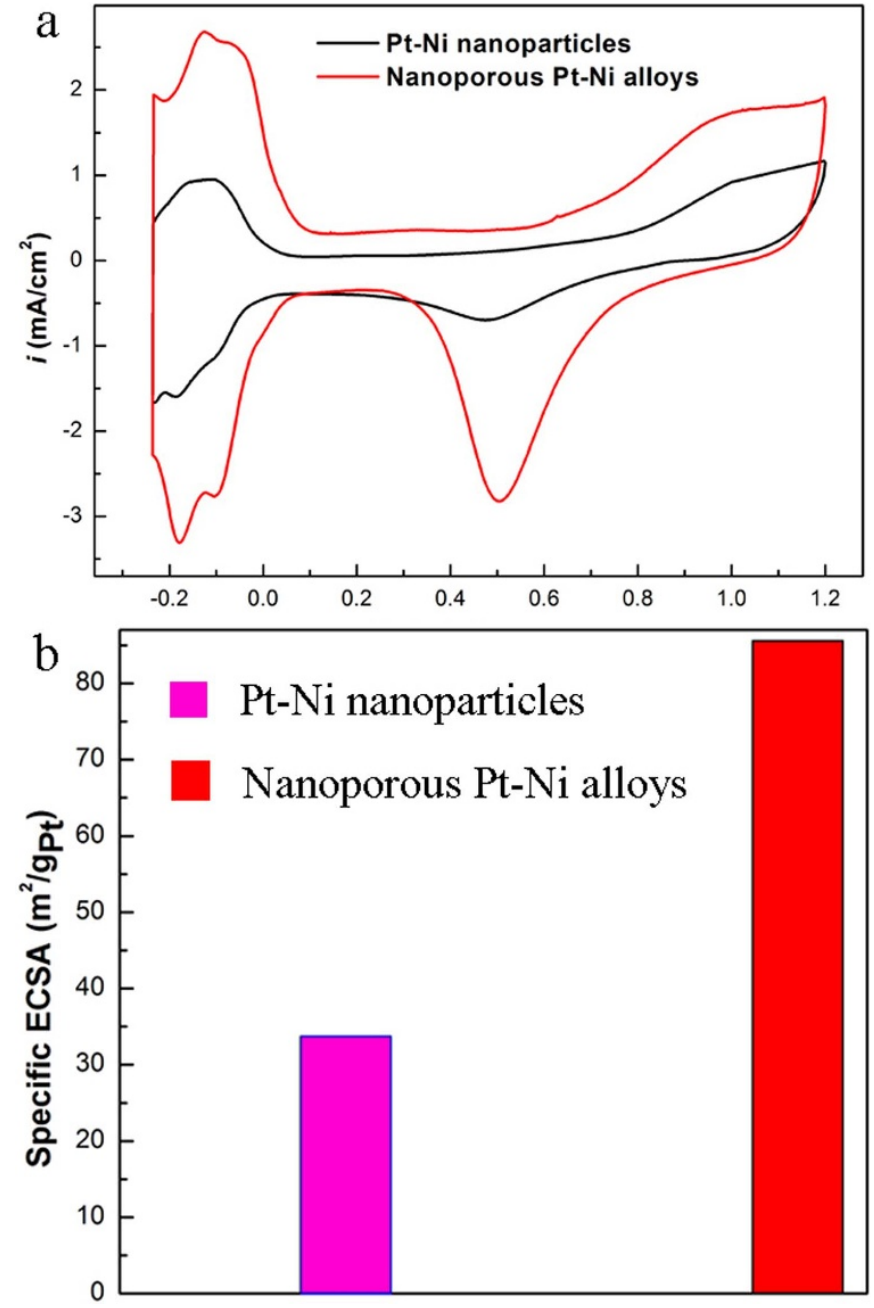

Figure 3 Electrochemical studies of alloys. (a) CV curves for Pt-Ni nanoparticles and nanoporous Pt-Ni alloys. (b) Specific ECSAs for Pt-Ni nanoparticles and nanoporous Pt-Ni alloys.

well dispersed in nonpolar solvents due to their hydrophobic surfaces, they can be regarded as "semi-homogeneous catalyst" which combines the advantages of both homogeneous and heterogeneous catalysts. The nanoparticles in solution phase are heterogeneous in nature. However, similar to homogeneous catalysts, they can be well dispersed in the solvent to facilitate the access of reactant molecules. Therefore, the novel nanocatalysts show considerable promise in semi-homogeneous catalysis.

Our chemical dealloying method holds at least three advantages in producing alloy nanoporous nanoparticles: (1) the procedure is very convenient and can finish within $1 \mathrm{~min}$, indicating that the production can be scaled up easily once one of them finds practical applications in catalysis; (2) because nanocrystalline alloys are used as precursors, the as-prepared dealloyed products show large surface area and porous characteristic, which are advantageous in their catalytic applications; and (3) it is a general strategy, which means that if we can get the nanocrystals of any alloys, corresponding nanoporous alloys can be prepared via this route. Fortunately, the methodology of synthesizing nanocrystalline alloys has been greatly developed by our group $^{31-33}$ and some other groups ${ }^{7-10,37-38}$ in recent years, so we can prepare a large variety of alloy nanoporous nanoparticles. Some examples are shown in Figure S4 (Supporting Information). Figure S4a and S4b exhibit TEM images of as-synthesized Pt-Co nanoparticles and Pt-Co nanoporous nanoparticles respectively. Their HRTEM images (Fig. S5) reveal clear lattice fringes with interplanar 
distances of about $0.21 \mathrm{~nm}$ and $0.22 \mathrm{~nm}$ respectively, which are characteristic d-spacings of cubic Pt-Co alloys in the (111) plane (the characteristic d-spacings of cubic Pt and cubic Co in the (111) plane are $0.23 \mathrm{~nm}$ and $0.20 \mathrm{~nm}$ respectively). XRD (Fig. S6) measurements confirm that, after corrosion, all the peaks are shifted slightly to smaller $2 \theta$ values (d-spacing increases), which is consistent with HRTEM results. EDS test (Fig. S7) indicates that the compositions of Pt-Co nanoparticles and nanoporous $\mathrm{Pt}$-Co are nearly $\mathrm{Pt}_{0.33} \mathrm{Ni}_{0.67}$ and $\mathrm{Pt}_{0.77} \mathrm{Ni}_{0.23}$ respectively. Figure $\mathrm{S} 4 \mathrm{c} \sim \mathrm{f}$ and Figure $\mathrm{S} 8 \sim 11$ show the results of $\mathrm{Pt}-\mathrm{Cu}$ and $\mathrm{Rh}-\mathrm{Ni}$ nanoparticles as well as nanoporous $\mathrm{Pt}-\mathrm{Cu}$ and $\mathrm{Rh}-\mathrm{Ni}$.

In summary, this work presents a successful synthesis of monodisperse alloy nanoporous nanoparticles via a facile chemical dealloying process using nanocrystalline alloys as precursors. The as-prepared alloy nanoporous nanoparticles have large surface area and small pores. The primary electrochemical tests confirm that alloy nanoporous nanoparticles show superior catalytic properties compared with alloyed nanoparticles. Our methodology provides a simple and convenient route to a variety of alloy nanoporous nanoparticles which represent a new class of nanomaterials with extraordinary structural characteristics. It is believed that these novel alloy nanoporous nanoparticles would open up new opportunities for catalytic applications.

\section{Methods}

Chemicals. All the reagents used in this work, including various kinds of metal inorganic salts, ODA, ethanol, cyclohexane, and nitric acid were of analytical grade from the Beijing Chemical Factory of China and were used without further purification.

Synthesis. $10 \mathrm{ml}$ of ODA was added into $50 \mathrm{ml}$ of beaker. The resulting solution was heated up to $120{ }^{\circ} \mathrm{C}$. Then, $0.1 \mathrm{mmol}$ of $\mathrm{H}_{2} \mathrm{PtCl}_{6} \cdot 6 \mathrm{H}_{2} \mathrm{O}$ and $0.2 \mathrm{mmol}$ of $\mathrm{Ni}\left(\mathrm{NO}_{3}\right)_{2} \cdot 6 \mathrm{H}_{2} \mathrm{O}$ were added. After the reactants were dissolved, the system was heated up to $220{ }^{\circ} \mathrm{C}$. The mixture was magnetically stirred for $10 \mathrm{~min}$ at this temperature. After reaction, Pt-Ni nanoparticles were collected at the bottom of the beaker. Then, the as-prepared Pt-Ni nanoparticles were added into $20 \mathrm{ml}$ of beaker, followed by the addition of an excess amount of concentrated nitric acid. With ultrasonic treatment for $1 \mathrm{~min}$ at room temperature, nanoporous $\mathrm{Pt}-\mathrm{Ni}$ alloys were collected at the bottom of the beaker. The collected products were washed several times with ethanol.

Characterization. The powder XRD patterns were recorded with a Bruker D8advance $\mathrm{X}$-ray powder diffractometer with $\mathrm{CuK} \alpha$ radiation $(\lambda=1.5406 \AA)$. The size and morphology of as-synthesized samples were determined by using Hitachi model H-800 transmission electron microscope and JEOL-2010F high-resolution transmission electron microscope. Energy dispersive spectroscopy was recorded to determine the composition of the products. BET surface areas and BJH pore-size distributions were measured with $\mathrm{N}_{2}$ at $77 \mathrm{~K}$ by using a Quantachrome Autosorb-1 instrument.

Electrochemical studies. Electrochemical measurements were conducted by using a three-compartment electrochemical cell. A saturated $\mathrm{Ag} / \mathrm{AgCl}$ electrode and a $\mathrm{Pt}$ wire were used as reference and counter electrodes, respectively. $\mathrm{HClO}_{4}$ was used as electrolyte. All cyclic voltammograms were recorded after 500 potential cycles and no change in voltammetric features was observed before and after electrochemical characterization.

1. Raney, M. Method of producing finely divided nickel. U.S. Patent 1628190 (1927).

2. Lee, H., Habas, S. E., Somorjai, G. A. \& Yang, P. Localized Pd overgrowth on cubic Pt nanocrystals for enhanced electrocatalytic oxidation of formic acid. J. Am. Chem. Soc. 130, 5406-5407 (2008).

3. Xu, D., Liu, Z. P., Yang, H. Z., Liu, Q. S., Zhang, J., Fang, J. Y., Zou, S. Z. \& Sun, K. Solution-based evolution and enhanced methanol oxidation activity of monodisperse Platinum-Copper nanocubes. Angew. Chem. Int. Ed. 48, 42174221 (2009).

4. Peng, Z. \& Yang, H. Designer platinum nanoparticles: control of shape, composition in alloy, nanostructure and electrocatalytic property. Nano Today 4 , 143-164 (2009).

5. Lim, B., Jiang, M., Camargo, P. H. C., Cho, E. C., Tao, J., Lu, X., Zhu, Y. \& Xia, Y. $\mathrm{Pd}-\mathrm{Pt}$ bimetallic nanodendrites with high activity for oxygen reduction. Science 324, 1302-1305 (2009).

6. Suryanarayana, C. Mechanical alloying and milling. Prog. Mater. Sci. 46, 1-184 (2001).
7. Sun, S. H., Murray, C. B., Weller, D., Folks, L. \& Moser, A. Monodisperse FePt nanoparticles and ferromagnetic FePt nanocrystal superlattices. Science 287, 1989-1992 (2000).

8. Wang, L. L. \& Johnson, D. D. Predicted trends of core-shell preferences for 132 late transition-metal binary-alloy nanoparticles. J. Am. Chem. Soc. 131, 1402314029 (2009)

9. Liu, Z. F., Jackson, G. S. \& Eichhorn, B. W. PtSn intermetallic, core-shell, and alloy nanoparticles as $\mathrm{CO}$-tolerant electrocatalysts for $\mathrm{H}_{2}$ oxidation. Angew. Chem. Int. Ed. 49, 3173-3176 (2010).

10. Yan, J. M., Zhang, X. B., Akita, T., Haruta, M. \& Xu, Q. One-step seeding growth of magnetically recyclable Au@Co core-shell nanoparticles: highly efficient catalyst for hydrolytic dehydrogenation of ammonia borane J. Am. Chem. Soc. 132, 53265327 (2010).

11. Snyder, J., Asanithi, P., Dalton, A. B. \& Erlebacher, J. Stabilized nanoporous metals by dealloying ternary alloy precursors. Adv. Mater. 20, 4883-4886 (2008).

12. Polarz, S. \& Smarsly, B. Nanoporous materials. J. Nanosci. Nanotechnol. 2, 581612 (2002).

13. Ding, Y. \& Chen, M. Nanoporous metals for catalytic and optical applications. MRS Bull. 34, 569-576 (2009).

14. Weissmuller, J., Newman, R. C., Jin, H. J., Hodge, A. M. \& Kysar, J. W. Nanoporous metals by alloy corrosion: formation and mechanical properties. MRS Bull. 34, 577-586 (2009).

15. Srivastava, R., Mani, P., Hahn, N. \& Strasser, P. Efficient oxygen reduction fuel cell electrocatalysis on voltammetrically dealloyed Pt-Cu-Co nanoparticles. Angew. Chem. Int. Ed. 46, 8988-8991 (2007).

16. Lai, F. J., Su, W. N., Sarma, L. S., Liu, D. G., Hsieh, C. A., Lee, J. F. \& Hwang, B. J. Chemical dealloying mechanism of bimetallic Pt-Co nanoparticles and enhancement of catalytic activity toward oxygen reduction. Chem. Eur. J. 16, 4602-4611 (2010).

17. Zhang, Q., Cobley, C. M., Zeng, J., Wen, L. P., Chen, J. \& Xia, Y. Dissolving Ag from Au-Ag alloy nanoboxes with $\mathrm{H}_{2} \mathrm{O}_{2}$ : a method for both tailoring the optical properties and measuring the $\mathrm{H}_{2} \mathrm{O}_{2}$ concentration. J. Phys. Chem. C 114, 63966400 (2010).

18. Xu, C., Su, J., Xu, X., Liu, P., Zhao, H., Tian, F. \& Ding, Y. Low temperature CO oxidation over unsupported nanoporous gold. J. Am. Chem. Soc. 129, 42-43 (2007).

19. Cobley, C. M., Campbell, D. J. \& Xia, Y. N. Tailoring the optical and catalytic properties of gold-silver nanoboxes and nanocages by introducing palladium. Adv. Mater. 20, 748-752 (2008).

20. Liu, L. F., Pippel, E., Scholz, R. \& Gosele, U. Nanoporous Pt-Co alloy nanowires: fabrication, characterization, and electrocatalytic properties. Nano Lett. 9, 43524358 (2009).

21. Liu, L. F., Scholz, R., Pippel, E. \& Gosele, U. Microstructure, electrocatalytic and sensing properties of nanoporous $\mathrm{Pt}_{46} \mathrm{Ni}_{54}$ alloy nanowires fabricated by mild dealloying. J. Mater. Chem. 20, 5621-5627 (2010).

22. Snyder, J., Fujita, T., Chen, M. W. \& Erlebacher, J. Oxygen reduction in nanoporous metal-ionic liquid composite electrocatalysts. Nature Mater. 9, 904907 (2010).

23. Huczko, A. Template-based synthesis of nanomaterials. Appl. Phys. A 70, 365-376 (2000).

24. Liu, X., Wang, A., Wang, X., Mou, C. Y. \& Zhang, T. Au-Cu alloy nanoparticles confined in SBA-15 as a highly efficient catalyst for CO oxidation. Chem. Commun. 3187-3189 (2008).

25. Wang, D., Xin, H. L., Yu, Y., Wang, H., Rus, E., Muller, D. A. \& Abruna, H. D. Ptdecorated PdCo@Pd/C core-shell nanoparticles with enhanced stability and electrocatalytic activity for the oxygen reduction reaction. J. Am. Chem. Soc. 132, 17664-17666 (2010).

26. Jiang, H. L., Akita, T., Ishida, T., Haruta, M. \& Xu, Q. Synergistic catalysis of Au@ Ag core-shell nanoparticles stabilized on metal-organic framework. J. Am. Chem. Soc. 133, 1304-1306 (2011).

27. Erlebacher, J., Aziz, M. J., Karma, A. \& Sieradzki, K. Evolution of nanoporosity in dealloying. Nature 410, 450-453 (2001).

28. Newman, R. C. \& Sieradzki, K. Corrosion science. MRS Bull. 24, 12-13 (1999).

29. Pugh, D. V., Dursun, A. \& Corcoran, S. G. Formation of nanoporous platinum by selective dissolution of $\mathrm{Cu}$ from $\mathrm{Cu}_{0.75} \mathrm{Pt}_{0.25}$. J. Mater. Res. 18, 216-221 (2003).

30. Cortie, M. B., Maaroof, A. I. \& Smith, G. B. Electrochemical capacitance of mesoporous gold. Gold Bull. 38, 14-22 (2005).

31. Wang, D. S. \& Li, Y. D. One-pot protocol for Au-based hybrid magnetic nanostructures via a noble-metal-induced reduction process. J. Am. Chem. Soc. 132, 6280-6281 (2010).

32. Wang, D. S., Peng, Q. \& Li, Y. D. Nanocrystalline intermetallics and alloys. Nano Res. 3, 574-580 (2010).

33. Wang, D. S. \& Li, Y. D. Bimetallic nanocrystals: liquid-phase synthesis and catalytic applications. Adv. Mater. 23, 1044-1060 (2011).

34. Pickering, H. W. \& Kim, Y. S. Dealloying at elevated-temperatures and at $298 \mathrm{~K}$ similarities and differences. Corros. Sci. 22, 621-635 (1982).

35. Shao, M. H., Shoemaker, K., Peles, A., Kaneko, K. \& Protsailo, L. Pt mono layer on porous Pd-Cu alloys as oxygen reduction electrocatalysts. J. Am. Chem. Soc. 132, 9253-9255 (2010).

36. Dutta, I., Carpenter, M. K., Balogh, M. P., Ziegelbauer, J. M., Moylan, T. E., Atwan, M. H. \& Irish, N. P. Electrochemical and structural study of a chemically dealloyed PtCu oxygen reduction catalyst. J. Phys. Chem. C 114, 16309-16320 (2010). 
37. Huang, X., Zhang, H., Guo, C., Zhou, Z. \& Zheng, N. Simplifying the creation of hollow metallic nanostructures: one-pot synthesis of hollow Palladium/Platinum single-crystalline nanocubes. Angew. Chem. Int. Ed. 48, 4808-4812 (2009).

38. Ding, Y., Fan, F., Tian, Z. \& Wang, Z. L. Atomic structure of Au-Pd bimetallic alloyed nanoparticles. J. Am. Chem. Soc. 132, 12480-12486 (2010).

\section{Acknowledgements}

This work was supported by the State Key Project of Fundamental Research for Nanoscience and Nanotechnology (2011CB932401, 2011CBA00500), NSFC (20921001, 21051001) and China Postdoctoral Science Foundation (20100470335).

\section{Author contributions}

D.W. performed the experiments, collected and analyzed the data, and wrote the paper; P.Z. helped with data analysis; Y.L. conceived the experiments, analyzed results, and wrote the paper.

\section{Additional information}

Supplementary Information accompanies this paper at http://www.nature.com/ scientificreports

Competing financial interests: The authors declare that they have no competing financial interests.

License: This work is licensed under a Creative Commons

Attribution-NonCommercial-ShareAlike 3.0 Unported License. To view a copy of this license, visit http://creativecommons.org/licenses/by-nc-sa/3.0/

How to cite this article: Wang, D., Zhao, P. \& Li, Y. General preparation for Pt-based alloy nanoporous nanoparticles as potential nanocatalysts. Sci. Rep. 1, 37; DOI:10.1038/ srep00037 (2011). 